\title{
MicroRNA-129-5p suppresses cell proliferation, migration and invasion via targeting ROCK1 in osteosarcoma
}

\author{
CHAOFAN HAN and WENBO WANG \\ Department of Orthopedic Surgery, First Affiliated Hospital of Harbin Medical University, \\ Harbin, Heilongjiang 150001, P.R. China
}

Received August 5, 2016; Accepted July 18, 2017

DOI: $10.3892 / \mathrm{mmr} .2018 .8374$

\begin{abstract}
Osteosarcoma (OS) is the most common primary malignancy of the bone in teenagers and accounts for $20-35 \%$ of all malignant primary bone tumors. Increasing evidence shows that microRNAs (miRNAs) are abnormally expressed in several types of human cancer. miRNAs are necessary to maintain the malignant phenotype of cancer cells and can function as either tumor suppressors or oncogenes. The present study aimed to measure the expression of miRNA-129-5p (miR-129-5p) in OS, determine the effects of miR-129-5p on the malignant behaviors of OS cells, and elucidate the molecular mechanism underlying the oncogenesis and progression of OS. The expression levels of miR-129-5p in OS tissues and cell lines were measured using reverse transcription-quantitative polymerase chain reaction (RT-qPCR) analysis. SAOS-2 and U2OS cells were then transfected with miR-129-5p mimics or miR-negative control. The effects of miR-129-5p on the proliferation, migration and invasion of SAOS-2 and U2OS cells in vitro were then evaluated using 3-(4,5-dimethylthiazol-2-yl)-2,5-diphenyltetrazolium bromide, Transwell migration assay and invasion assays, respectively. In addition, bioinformatics analysis, a luciferase reporter assay, and RT-qPCR and western blot analyses were used to examine whether Rho-associated protein kinase 1 (ROCK1) was a direct target of miR-129-5p. The mRNA expression of ROCK1 in OS tissues was detected using RT-qPCR analysis, and the biological roles of ROCK1 in OS cells were also evaluated. The results showed that miR-129-5p was significantly downregulated in the OS tissues and cell lines. The re-expression of miR-129-5p suppressed the cell proliferation, migration and invasion of OS cells. In addition, ROCK1 was confirmed as a direct target of miR-129-5p. The mRNA expression of
\end{abstract}

Correspondence to: Professor Wenbo Wang, Department of Orthopedic Surgery, First Affiliated Hospital of Harbin Medical University, 23 Youzheng Street, Nangang, Harbin, Heilongjiang 150001, P.R. China

E-mail: wenbo_wang23@163.com

Key words: microRNA-129-5p, osteosarcoma, Rho-associated protein kinase 1 , invasion, proliferation, migration
ROCK1 was high in OS tissues and inversely correlated with the expression of miR-129-5p. The downregulation of ROCK1 inhibited the proliferation, migration and invasion of OS cells. These findings suggested that miR-129-5p inhibited cell proliferation, migration and invasion in the development of OS via the negative regulation of ROCK1. The miR-129-5p/ROCK1 axis may serve as an efficient target in cancer therapy.

\section{Introduction}

Osteosarcoma is the most common primary malignancy of bone in teenagers and accounts for $20-35 \%$ of all malignant primary bone tumors $(1,2)$. It originates from primitive transformed cells of mesenchymal origin in bone, which exhibit osteoblastic differentiation and produce malignant osteoid (3). Currently, the most common treatments for patients with OS are neoadjuvant chemotherapy, surgery and adjuvant chemotherapy (4). With development in the therapeutic strategies, the 5 -year overall and disease-free survival rates for patients with OS have improved in the last 30 years (5). However, the curative effects in the treatment of those patients with metastasis or at an advanced clinical stage have been less effective in this period (6). The molecular mechanisms underlying the occurrence and development of OS remain to be fully elucidated (7). Therefore, it is important to fully understand the mechanism underlying the metastasis and progression in OS, and to investigate therapeutic targets to improve the prognosis of this disease.

MicroRNAs (miRNAs) are an abundant class of non-coding, conserved, single-strand RNA molecules containing 18-25 nucleotides $(8,9)$. It is well established that miRNAs are important in several diverse biological processes, including cell proliferation, cell cycle, apoptosis, metastasis, invasion, angiogenesis, stress responses and differentiation (10). miRNAs exert their functions through interaction with the 3' untranslated regions (3'UTRs) of target genes in a base pairing manner, and mainly result in translational repression or target mRNA degradation $(11,12)$. At present, $>1,000$ miRNAs have been identified in the human genome, and these can modulate the expression of thousands of human protein-encoding genes $(9,13)$. Accumulated evidence shows that numerous miRNAs are abnormally expressed in several types of human cancer (14-16). These miRNAs are necessary to maintain the malignant phenotype of cancer cells, and can 
function as either tumor suppressors or oncogenes depending on the tumor type and effects of their target genes (17). This suggests there is merit in investigating miRNAs as novel and efficient therapeutic targets for antitumor therapy.

Previous studies have reported that miR-129-5p is involved in the carcinogenesis and progression of several types of human cancer, including breast cancer (18), gastric cancer (19), lung cancer (20), thyroid carcinoma (21), ovarian cancer (22) and colorectal cancer (23). However, the expression, effects and mechanisms underlying the effects of miR-129-5p in OS remain to be elucidated. Therefore, in the present study, the expression levels of miR-129-5p in OS tissues and cell lines were examined, and the biological roles of the direct target genes of miR-129-5p in OS were investigated.

\section{Materials and methods}

Cell lines and tumor specimens. The human OS cell lines, MG-63, SAOS-2, HOS, 143B and U2OS, were purchased from American Type Culture Collection (Manassas, VA, USA). A human normal osteoblastic cell line (hFOB 1.19) was also obtained from American Type Culture Collection. The cells were grown in Dulbecco's modified Eagle's medium (Gibco; Thermo Fisher Scientific, Inc., Waltham, MA, USA) containing $10 \%$ fetal bovine serum (FBS) and $1 \%$ penicillin/streptomycin (Invitrogen; Thermo Fisher Scientific, Inc.). The cultures were maintained at $37^{\circ} \mathrm{C}$ in a $5 \% \mathrm{CO}_{2}$ atmosphere. In addition, 19 pairs of OS tissues and corresponding adjacent normal bone tissues (13 male, 6 female; age range 23-67 years; mean age, 42 years; I+IIA stage, 11; IIB/III stage, 8) were collected from the Department of Orthopedic Surgery, First Affiliated Hospital of Harbin Medical University (Harbin, China) between August 2013 to December 2015. The present study was approved by the Ethics Committee of the First Affiliated Hospital of Harbin Medical University.

Reverse transcription-quantitative polymerase chain reaction $(R T-q P C R)$ analysis. Total RNA was extracted using TRIzol ${ }^{\circledR}$ reagent (Invitrogen; Thermo Fisher Scientific, Inc.) according to the manufacturer's protocol. The NanoDrop ND-1000 spectrophotometer (Thermo Fisher Scientific, Inc.) was used to measure the concentration. Reverse transcription was performed using M-MLV (Promega Corporation, Madison, WI, USA). The temperature protocol for reverse transcription was as follows: $95^{\circ} \mathrm{C}$ for $2 \mathrm{~min} ; 20$ cycles of $94^{\circ} \mathrm{C}$ for $1 \mathrm{~min}, 55^{\circ} \mathrm{C}$ for $1 \mathrm{~min}$ and $72^{\circ} \mathrm{C}$ for $2 \mathrm{~min}$; then $72^{\circ} \mathrm{C}$ for $5 \mathrm{~min}$. The expression levels of miR-129-5p and the mRNA expression levels of Rho-associated protein kinase 1 (ROCK1) were determined using SYBR Premix Ex Taq ${ }^{\mathrm{TM}}$ kits (Takara Bio, Inc., Tokyo, Japan). The reaction system contained $10 \mu \mathrm{l}$ SYBR Premix Ex Taq, $2 \mu \mathrm{l}$ cDNA (200 ng), $0.8 \mu$ l forward primer, $0.8 \mu \mathrm{l}$ reverse primer, $0.4 \mu \mathrm{l}$ ROX reference dye and $6 \mu \mathrm{ldd} \mathrm{H}_{2} \mathrm{O}$. The amplification was performed with cycling conditions as follows: $5 \mathrm{~min}$ at $95^{\circ} \mathrm{C}$, followed by 40 cycles of $95^{\circ} \mathrm{C}$ for $30 \mathrm{sec}$ and $65^{\circ} \mathrm{C}$ for $45 \mathrm{sec}$. GAPDH and U6 were used as the internal reference for ROCK1 mRNA and miR-129-5p, respectively. RT-qPCR analysis was performed on an Applied Biosystems ${ }^{\circledR} 7900$ HT Real-Time PCR system (Thermo Fisher Scientific, Inc.). The primers were designed as follows: miR-129-5p, 5'-GATACTCACTTTTTGCGGTCT-3' (forward) and 5'-GTGCAGGGTCCGAGGT-3' (reverse); U6, 5'-CTCGCTTCGGCAGCACA-3' (forward) and 5'-AAC GCTTCACGAATTTGCGT-3' (reverse); ROCK1, 5'-ATG AGTTTATTCCTACACTCTACCACTTTC-3' (forward) and 5'-TAACATGGCATCTTCGACACTCTAG-3' (reverse); and GAPDH, 5'-ACACCCACTCCTCCACCTTT-3' (forward) and 5'-TAGCCAAATTCGTTGTCATACC-3' (reverse). Data were calculated and normalized using the $2^{-\Delta \Delta \mathrm{Cq}}$ method (24).

Transfection of cells. The miR-129-5p mimics and negative control miRNA mimics (miR-NC) were synthesized by GenePharma (Shanghai, China). Small interfering (si)RNA targeting ROCK1 (si-ROCK1) and siRNA negative control (si-NC) were purchased from Biomics Biotechnologies Co., Ltd. (Nantong, China). The si-ROCK1 sequence was 5'-GGG UAACUCAUCUGGUAAATT-3' and the si-NC sequence was 5'-UUCUCCGAACGUGUCACGUTT-3'. The cells were seeded into 6-well plates at a density of 50-60\% 1 day prior to transfection. Cell transfection was performed using Lipofectamine 2000 (Invitrogen; Thermo Fisher Scientific, Inc.) according to the manufacturer's protocol.

3-(4,5-dimethylthiazol-2-yl)-2,5-diphenyltetrazolium bromide (MTT) assay. Cell proliferation was evaluated using an MTT assay (Sigma-Aldrich; Merck Millipore, Darmstadt, Germany) according to the manufacturer's protocol. Briefly, cells grown in the logarithmic phase were seeded in 96-well plates at a density of 2,500 cells/well. Following adherence, the cells were transfected with the miRNA mimics or siRNA, followed by incubation at $37^{\circ} \mathrm{C}$ in $5 \% \mathrm{CO}_{2}$ atmosphere for $24,48,72$ and $96 \mathrm{~h}$. The MTT assay was then performed at each time point; $20 \mu \mathrm{l}$ MTT solution was added to the culture medium and incubated at $37^{\circ} \mathrm{C}$ for an additional $4 \mathrm{~h}$. Subsequently, the MTT formazan was dissolved by $150 \mu 1$ DMSO (Sigma-Aldrich; Merck Millipore). The absorbance was determined at $490 \mathrm{~nm}$ using an automatic multi-well spectrophotometer (Bio-Rad Laboratories, Inc., Hercules, CA, USA).

Transwell migration and invasion assays. The cell migration and invasion capacities were assessed using Transwell chambers ( $8-\mu \mathrm{m}$ pores; BD Biosciences, San Jose, CA, USA). For the invasion assay, the chambers were coated with $60 \mu \mathrm{l}$ of Matrigel (5 mg/ml; BD Biosciences). The cells were collected $48 \mathrm{~h}$ following transfection and, at a density of $4 \times 10^{4}$ in $200 \mu \mathrm{l}$ FBS-free medium, were seeded in the upper chamber. Culture medium $(600 \mu \mathrm{l})$ supplemented with $20 \%$ FBS was added to the lower chamber. Following incubation at $37^{\circ} \mathrm{C}$ in a $5 \%$ $\mathrm{CO}_{2}$ atmosphere for $48 \mathrm{~h}$, cotton swabs were used to wipe the cells remaining in the upper chamber. The invaded cells were stained with $0.5 \%$ crystal violet for $30 \mathrm{~min}$ and then counted in five randomly selected fields under a light microscope (magnification, x200; Olympus Corporation, Tokyo, Japan). For the migration assay, the procedures were similar to those in the invasion assay, with the exception that the upper chambers were not coated with Matrigel.

miRNA target prediction and luciferase reporter assay. To determine the potential target genes of miR-129-5p, bioinformatics analysis was performed with TargetScan 7.0 (http://www.targetscan.org/)andmiRanda(August2010release; 
http://www.microrna.org). psiCHECK ${ }^{\mathrm{TM}}$ 2-ROCK1-wild-type (WT)-3'UTR and psiCHECK ${ }^{\mathrm{TM}} 2$-ROCK1-mutant (MUT)-3'UTR were synthesized by GenePharma. At 1 day prior to transfection, HEK293T cells (American Type Culture Collection) were seeded in 24-well plates at a density of $1 \times 10^{5}$ cells/well. psiCHECK ${ }^{\text {TM }} 2$-ROCK1-WT-3'UTR or psiCHECK ${ }^{\mathrm{TM}}$ 2-ROCK1-MUT-3'UTR, together with the miR-129-5p mimics or miR-NC were transfected into the HEK293T cells using Lipofectamine 2000. The culture medium was replaced with DMEM containing $10 \% \mathrm{FBS}$ at $8 \mathrm{~h}$ post-transfection. The cells were incubated at $37^{\circ} \mathrm{C}$ in $5 \% \mathrm{CO}_{2}$ atmosphere for $48 \mathrm{~h}$ and luciferase activities were determined using a Dual-Luciferase Reporter Assay system (Promega Corporation). Renilla luciferase activities were normalized to firefly luciferase activities.

Western blot analysis. Total protein was isolated from the tissues and cell lines using RIPA buffer (Santa Cruz Biotechnology, Inc., Dallas, TX, USA) supplemented with protease inhibitors and phosphate inhibitors (Roche Diagnostics, Indianapolis, IN, USA). A Bicinchoninic Acid Protein assay kit (Thermo Fisher Scientific, Inc.) was used to detect protein concentrations. Equal quantities of protein $(30 \mu \mathrm{g})$ were separated by $10 \%$ SDS-PAGE gel and transferred onto a polyvinylidene difluoride membrane (EMD Millipore, Bedford, MA, USA) followed by blocking with 5\% non-fat milk in Tris-buffered saline-Tween 20 (TBST) for $1 \mathrm{~h}$ at room temperature. The membranes were then immunoblotted with primary antibodies at $4^{\circ} \mathrm{C}$ overnight. Following washing three times with TBST, the membranes were incubated with goat anti-mouse horseradish peroxidase-conjugated secondary antibody (cat no. sc-2005; 1:2,000; Santa Cruz Biotechnology, Inc.) for $2 \mathrm{~h}$ at room temperature. Finally, immunoreactive protein bands were visualized using the enhanced chemiluminescence system (Pierce; Thermo Fisher Scientific, Inc.). The primary antibodies used in the present study were as follows: Mouse anti-human monoclonal ROCK1 antibody (cat no. sc-365628; 1:1,000) and mouse anti-human monoclonal GAPDH antibody (cat no. sc-47724; 1:1,000) (both from Santa Cruz Biotechnology, Inc.). The densitometry of protein bands was quantified using ImageJ version 1.49 (National Institutes of Health, Bethesda, MD, USA).

Statistical analysis. Data are presented as the mean \pm standard deviation. The differences between groups were compared using two-tailed Student's t-test or one-way analysis of variance using SPSS 19.0 statistical software (IBM SPSS Inc., Armonk, NY, USA). SNK analysis was performed as a post hoc test following analysis of variance. $\mathrm{P}<0.05$ was considered to indicate a statistically significant difference.

\section{Results}

miR-129-5p is downregulated in OS tissues and OS cell lines. The expression of miR-129-5p was determined in OS tissues and corresponding adjacent normal bone tissues using RT-qPCR analysis. As shown in Fig. 1A, the expression of miR-129-5p was significantly lower in the OS tissues, compared with that in the corresponding adjacent normal bone tissues $(\mathrm{P}<0.05)$. The expression levels of miR-129-5p
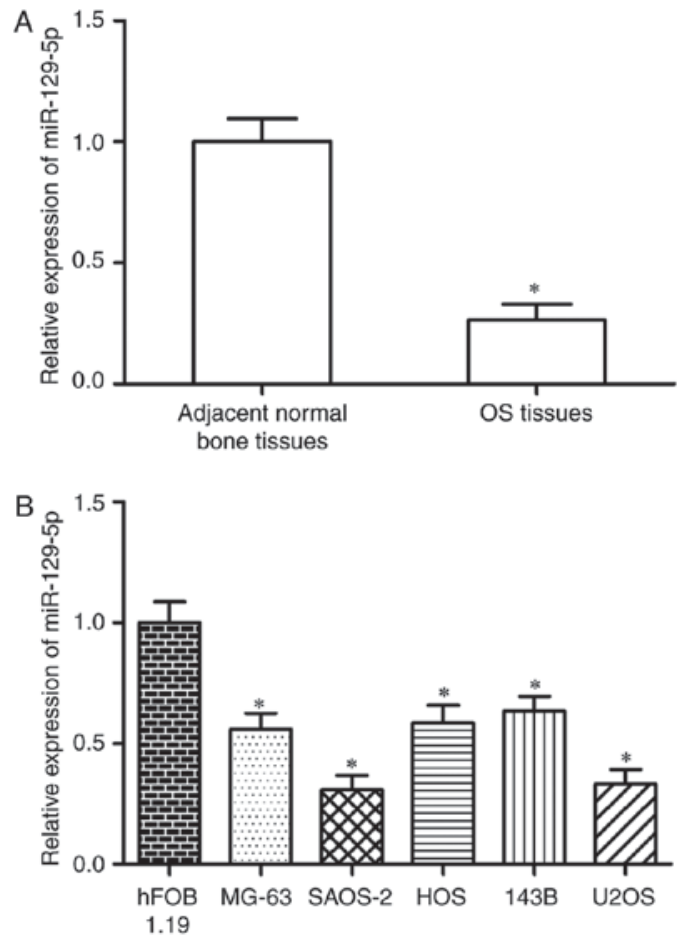

Figure 1. miR-129-5p is expressed at low levels in OS tissues and cell lines (A) Expression levels of miR-129-5p in OS tissues and corresponding adjacent normal bone tissues were determined using RT-qPCR analysis. (B) RT-qPCR analysis was performed to measure the expression of miR-129-5p in OS cell lines and a human normal osteoblastic cell line. ${ }^{*} \mathrm{P}<0.05$. OS, osteosarcoma; miR, microRNA; RT-qPCR, reverse transcription-quantitative polymerase chain reaction.

in the MG-63, SAOS-2, HOS, 143B and U2OS cells, and in the human normal osteoblast cell line (hFOB 1.19) were also detected. Compared with the expression in hFOB 1.19 cells, the expression of miR-129-5p was downregulated in all five OS cell lines (Fig. 1B; $\mathrm{P}<0.05$ ).

miR-129-5p inhibits cell proliferation, migration and invasion in $O S$. To examine the potential roles of miR-129-5p in OS, the SAOS-2 and U2OS cells were transfected with miR-129-5-mimics or miR-NC. Following transfection, the expression of miR-129-5p was significantly increased in the SAOS-2 and U2OS cells transfected with the miR-129-5p mimics (Fig. 2A; P<0.05). The MTT assay showed that cell proliferation was inhibited in the miR-129-5p mimic-transfected SAOS-2 and U2OS cells, compared with that in the miR-NC-transfected cells (Fig. 2B; $\mathrm{P}<0.05$ ). Similarly, the Transwell migration and invasion assays indicated that the ectopic expression of miR-129-5p decreased the migration and invasion capacities of the SAOS-2 and U2OS cells (Fig. 2C; $\mathrm{P}<0.05$ ). Taken together, these data suggested that miR-129-5p acted as a tumor suppressor in OS via inhibiting cell proliferation, migration and invasion.

ROCK1 is a direct target of miR-129-5p. To examine the molecular mechanism underlying the tumor suppressive roles of miR-129-5p in OS, bioinformatics analysis was performed using TargetScan 7.0 and miRanda. A number of genes were predicated as potential direct target genes of miR-129-5p, however, ROCK1 was selected for further analysis as it has 

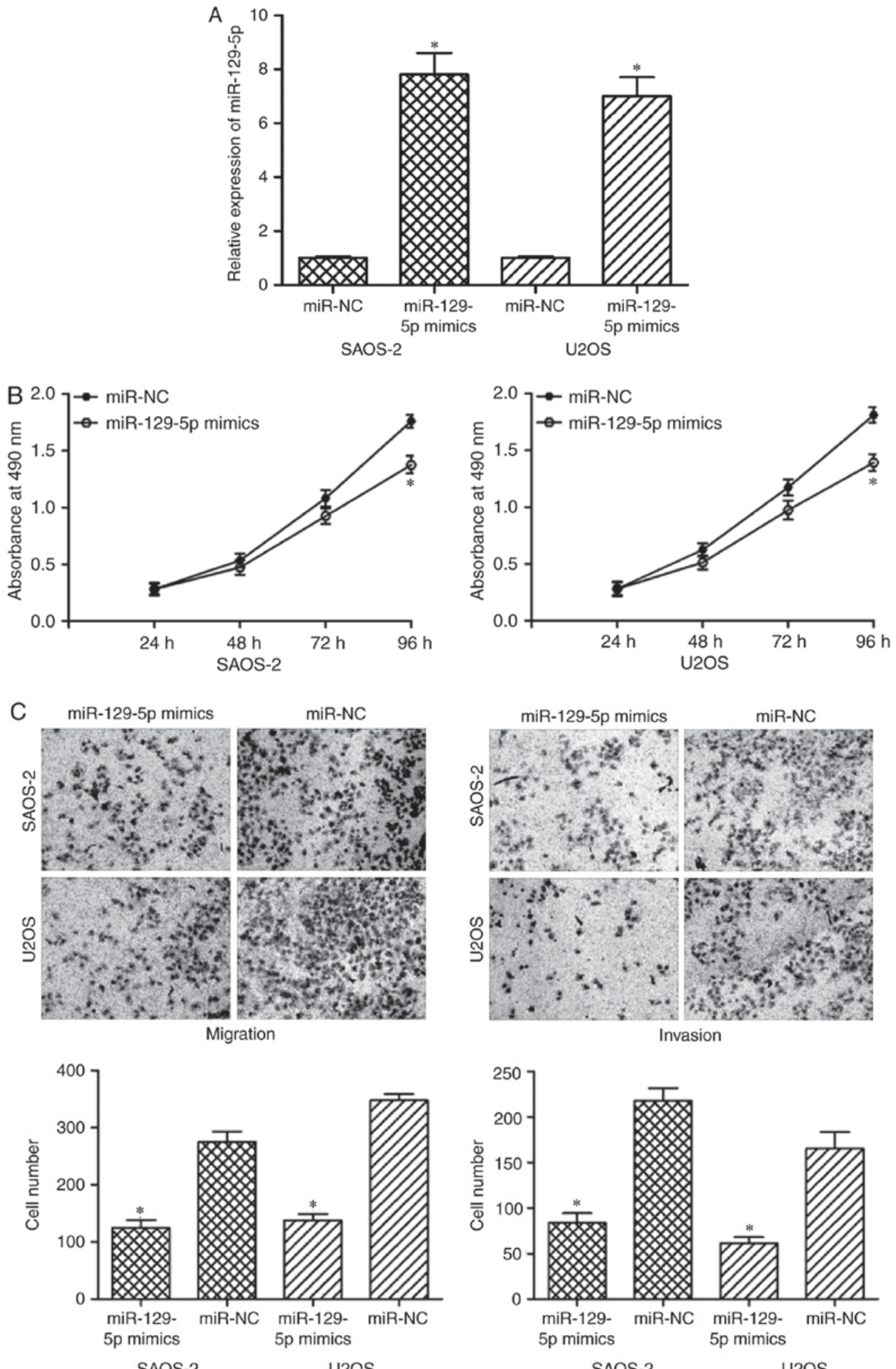

Figure 2. miR-129-5p suppresses the proliferation, migration and invasion of SAOS-2 and U2OS cells. (A) SAOS-2 and U2OS cells were transfected with miR-129-5p mimics or miR-NC. Expression of miR-129-5p was significantly upregulated in the SAOS-2 and U2OS cells following transfection with miR-129-5p mimics. (B) A 3-(4,5-dimethylthiazol-2-yl)-2,5-diphenyltetrazolium bromide assay was used to analyze the proliferation of SAOS-2 and U2OS cells transfected with miR-129-5p mimics or miR-NC. (C) Transwell migration and invasion assays were performed to examine the migration and invasion capacities of SAOS-2 and U2OS cells transfected with miR-129-5p mimics or miR-NC (magnification, x200). ${ }^{*} \mathrm{P}<0.05$, vs. miR-NC. miR, microRNA; NC, negative control.

been reported to be involved in the carcinogenesis and progression of OS (25) (Fig. 3A; $\mathrm{P}<0.05)$.

A luciferase reporter assay was then performed to investigate whether ROCK 1 is adirect target of miR-129-5p.HEK293T cells were co-transfected with psiCHECK ${ }^{\mathrm{TM}} 2$-ROCK1-WT-3'UTR or psiCHECK ${ }^{\mathrm{TM}}$ 2-ROCK1-MUT-3'UTR and miR-129-5p mimics or miR-NC. The results showed that the upregulation of miR-129-5p suppressed the luciferase activities 
A

\begin{tabular}{|c|c|c|}
\hline ROCK1-WT-3' UTR & $5^{\prime}$ & UUUCAAAAAAU... \\
\hline hsa-miR-129-5p & $3^{\prime}$ & CGUUCGGGUCUGGCGUUUUUC \\
\hline ROCK1-MUT-3' UTR & $5^{\prime}$ & ....UAAGUAUAGAAAUUUCUUUUUU... \\
\hline \multicolumn{3}{|c|}{ Target region 2} \\
\hline ROCK1-WT-3' UTR & $5^{\prime}$ & ...UGACUUUAUAAAAC \\
\hline sa-miR-129-5p & $3^{\prime}$ & CGUUCGGGUCL \\
\hline OCK1-MUT-3' UTR & $5^{\prime}$ & ...UGACUUUAUAAAACL \\
\hline
\end{tabular}
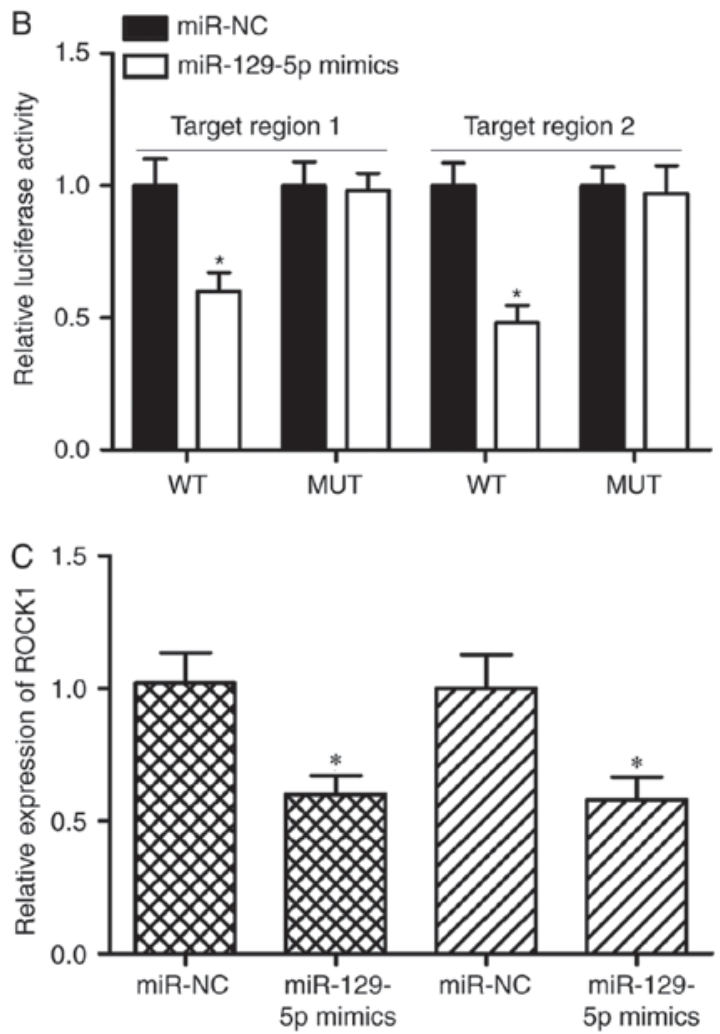

SAOS-2

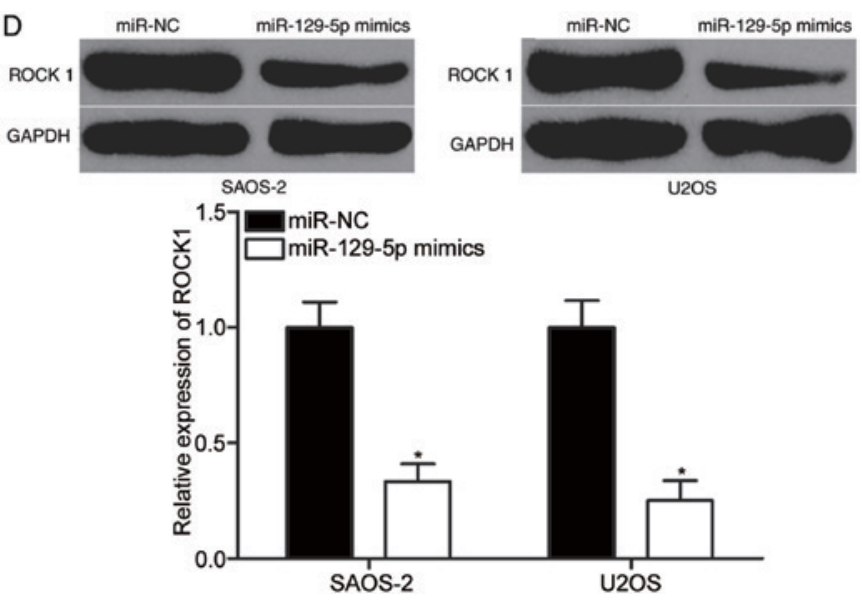

Figure 3. ROCK1 is a direct target of miR-129-5p. (A) Schematic illustration of two conserved miR-129-5p binding sites in the 3'UTR of ROCK1.(B) Luciferase activities were detected in HEK293T cells $48 \mathrm{~h}$ following co-transfection with psiCHECK $^{\text {TM }}$ 2-ROCK1-WT-3'UTR or psiCHECK ${ }^{\text {TM }}$ 2-ROCK1-MUT-3'UTR, and miR-129-5p mimics or miR-NC. (C) mRNA expression levels of ROCK1 in SAOS-2 and U2OS cells transfected with miR-129-5p mimics or miR-NC were detected using reverse transcription-quantitative polymerase chain reaction analysis. (D) Protein expression levels of ROCK1 were measured in SAOS-2 and U2OS cells transfected with miR-129-5p mimics or miR-NC. ${ }^{*} \mathrm{P}<0.05$, vs. miR-NC. miR, microRNA; ROCK1, Rho-associated protein kinase 1; 3'UTR, 3' untranslated region; NC, negative control; WT, wild-type; MUT, mutant.
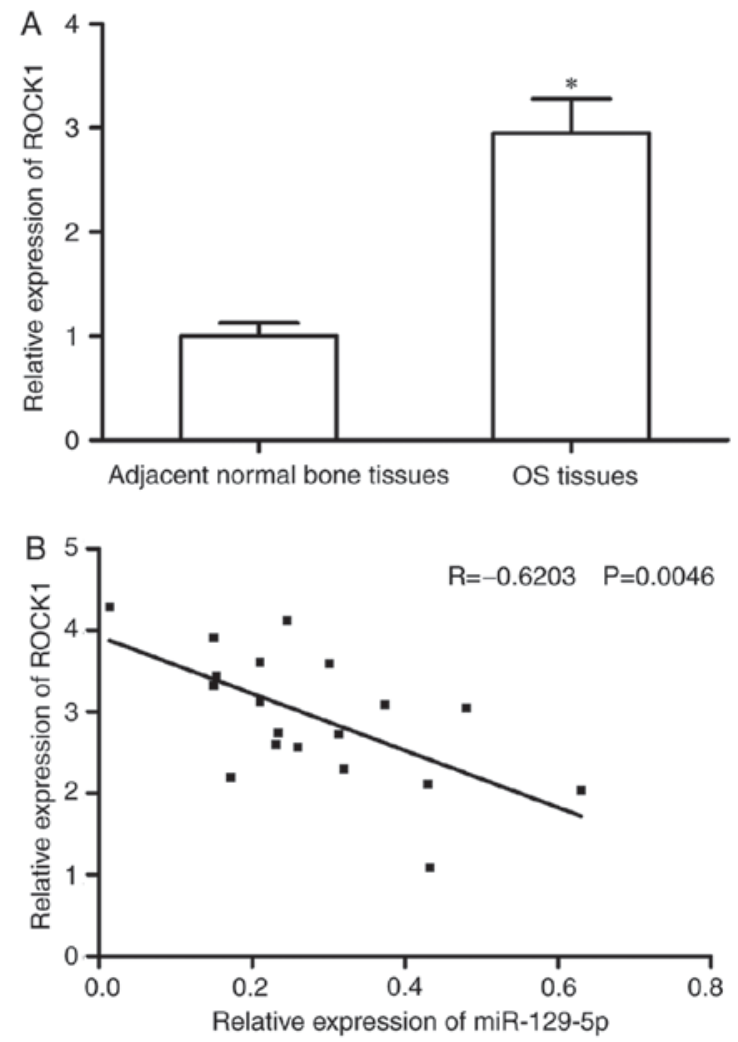

Figure 4. ROCK1 is upregulated in OS tissues and inversely correlated with the expression of miR-129-5p expression. (A) Relative mRNA expression of ROCK1 was determined in OS tissues and corresponding adjacent normal bone tissues using reverse transcription-quantitative polymerase chain reaction analysis. (B) Upregulation of ROCK1 in OS tissues was negatively correlated with the expression of miR-129-5p. ${ }^{*} \mathrm{P}<0.05$, vs. normal tissues. OS osteosarcoma; miR, microRNA; ROCK1, Rho-associated protein kinase 1.

of psiCHECK ${ }^{\text {TM }}$ 2-ROCK1-WT-3'UTR (Fig. 3B; P<0.05) but had no inhibitory effects on the luciferase activities of psiCHECK $^{\text {Tм }}$ 2-ROCK1-MUT-3'UTR, suggesting that ROCK1 may be a direct target of miR-129-5p.

To confirm the regulatory roles of miR-129-5p in the expression of ROCK1, the expression levels of ROCK1 were measured in SAOS-2 and U2OS cells following transfection with miR-129-5p mimic or miR-NC. The results of RT-qPCR and western blot analyses indicated that the mRNA (Fig. 3C; $\mathrm{P}<0.05$ ) and protein (Fig. 3D; $\mathrm{P}<0.05$ ) levels of ROCK1 were downregulated in the miR-129-5p mimic-transfected SAOS-2 and U2OS cells, compared with those in the miR-NC group. Taken together, these results suggested that ROCK1 may be a direct target of miR-129-5p.

ROCK1 is upregulated in OS tissues and inversely correlated with the expression levels of miR-129-5p. As the results demonstrated that ROCK1 was a direct target of miR-129-5p in OS, the present study detected the expression of ROCK1 in OS tissues and corresponding adjacent normal bone tissues using RT-qPCR analysis. The results showed that the mRNA expression of ROCK1 was significantly higher in the OS tissues, compared with that in corresponding adjacent normal bone tissues (Fig. 4A; $\mathrm{P}<0.05$ ). The associations between the expression of miR-129-5p and the mRNA expression of ROCK1 in OS tissues were analyzed using Spearman's 
correlation analysis, and it was found that the mRNA expression of ROCK1 was inversely correlated with the expression of miR-129-5p in OS tissues ( $\mathrm{R}=-0.6203 ; \mathrm{P}=0.0046$; Fig. 4B).

miR-129-5p triggers the inhibition of OS cell proliferation, migration and invasion, in part, via targeting ROCK1. To investigate whether the downregulation of ROCK1 contributed to the tumor suppressive roles of miR-129-5p in cell proliferation, migration and invasion, the biological functions of ROCK1 were determined using an RNA interference method. SAOS-2 and U2OS cells were injected with si-ROCK1 or si-NC. The results of the RT-qPCR and western blot analyses indicated that the mRNA (Fig. 5A; $\mathrm{P}<0.05$ ) and protein (Fig. $5 \mathrm{~B} ; \mathrm{P}<0.05$ ) expression levels of ROCK1 were reduced in the SAOS-2 and U2OS cells transfected with si-ROCK1. Subsequently, an MTT assay, and Transwell migration and invasion assays were performed to evaluate the roles of ROCK1 in OS. As shown in Fig. 5C and D, the downregulation of ROCK1 suppressed SAOS-2 and U2OS cell proliferation $(\mathrm{P}<0.05)$, migration and invasion $(\mathrm{P}<0.05)$. These findings demonstrated that the biological functions of the underexpression of ROCK1 were similar to those induced by the overexpression of miR-129-5p in OS cells, suggesting that the antitumor effect of miR-129-5p was, in part, dependent on the downregulation of ROCK1.

\section{Discussion}

miR-129 is transcribed from two genes, mir-129-1 and mir-129-2. The mature miRNAs, miR-129-5p and miR-129-3p, are processed from $5^{\prime}$ and $3^{\prime}$ precursors, respectively (26). In the present study, it was shown that miR-129-5p was downregulated in OS tissues and cell lines, compared with corresponding adjacent normal bone tissues and a human normal osteoblast cell line, respectively. In addition, the re-expression of miR-129-5p inhibited cell proliferation, migration and invasion in OS. ROCK1 was identified as a novel direct target gene of miR-129-5p, and miR-129-5p negatively regulated the expression of ROCK1 via binding to its 3 'UTR. Taken together, these findings suggested that miR-129-5p may be a novel potential therapeutic target for the treatment of patients with OS.

miR-129-5p has been found to be abnormally expressed in various types of cancer. For example, Yu et al (18) reported that the expression of miR-129-5p was lower in breast cancer tissues than paired adjacent normal breast tissues; the downregulation of miR-129-5p was significantly associated with advanced clinical stage and poor prognosis in patients with breast cancer. Jiang et al (19) found that the levels of miR-129-5p were reduced in gastric cancer tissues and blood samples, compared with those in matched non-tumor adjacent tissues and healthy volunteers, respectively. Ma et al (27) demonstrated that miR-129-5p was downregulated in hepatocellular carcinoma, and was correlated with vascular invasion, intrahepatic metastasis and recurrence rate. In addition, patients with hepatocellular carcinoma with a low expression of miR-129-5p had a poorer outcome, compared with patients with a high level of miR-129-5p. Similarly, Zhai et al (28) revealed that a low expression of miR-129-5p was correlated with advanced stage and vascular invasion. miR-129-5p has also been found to be expressed at low levels in lung cancer (20), thyroid carcinoma (21), ovarian cancer (22) and colorectal cancer (23). These findings suggest that miR-129-5p is involved in carcinogenesis and cancer progression.

Previous studies have reported that miR-129-5p functions as a tumor suppressor in several types of human cancer. For example, in breast cancer, the overexpression of miR-129-5p inhibits cell mobility and migration, reverses epithelial-mesenchymal transition, attenuates irradiation-induced autophagy and decreases radioresistance $(18,29,30)$. In gastric cancer, the upregulation of miR-129-5p causes marked suppression of cell viability, colony-forming ability, migration and invasion (19). Ma et al (27) showed that the ectopic expression of miR-129-5p suppressed cell growth, invasion and metastasis in hepatocellular carcinoma (28). Duan et al (21) reported that the re-expression of miR-129-5p reduced cell migration and induces apoptosis of thyroid carcinoma. Tan et al (22) demonstrated that the enforced expression of miR-129-5p repressed ovarian cancer cell proliferation, survival and tumorigenicity. In colorectal cancer, miR-129-5p has been shown to inhibit cell growth, induce apoptosis and enhance cell-cycle arrest in colorectal cancer (23). These findings indicate that miR-129-5p is important in these types of human cancer, and may serve as a potential therapeutic target for their treatment.

To investigate the potential molecular mechanism by which miR-129-5p regulates the proliferation and metastasis in OS, the present study performed bioinformatics analysis using two prediction programs. ROCK1 was predicted as a potential target gene of miR-129-5p. The experimental data further revealed that miR-129-5p suppressed the luciferase activities of the psiCHECK ${ }^{\mathrm{TM}}$ 2-ROCK1-WT-3'UTR reporter but did not affect that of a 'seed region' ROCK1-MUT-3'UTR reporter. In addition, the overexpression of miR-129-5p decreased the mRNA and protein expression of ROCK1 in OS cells. The mRNA expression of ROCK1 was expressed at high levels in OS tissues, compared with corresponding adjacent normal bone tissues, and its expression was inversely correlated with the expression level of miR-129-5p. The biological effects of the underexpression of ROCK1 were similar to those induced by the overexpression of miR-129-5p in OS cells, suggesting that the antitumor effect of miR-129-5p was, at least in part, dependent on the downregulation of ROCK1. These data suggested that miR-129-5p directly negatively regulated the expression of ROCK1 via binding to its $3^{\prime} \mathrm{UTR}$.

ROCK is an essential downstream effector of the Rho small GTPase and functions predominantly as a molecular switch, which binds GTP and GDP to modulate cell proliferation, survival and cytoskeleton organization, inducing changes in cell shape/morphology, invasion and movement (31-33). There is considerable and increasing evidence supporting the importance of the ROCK1 in the development and progression of OS. Liu et al (25) found that ROCK1 was significantly upregulated in OS tissues and cell lines. The high expression level of ROCK1 was correlated with poor prognosis in patients with OS, whereas the downregulation of ROCK1 suppressed cell proliferation and viability, and increased apoptosis in OS. Wang et al (34) indicated that the underexpression of ROCK1 repressed OS cell migration and invasion. These findings 

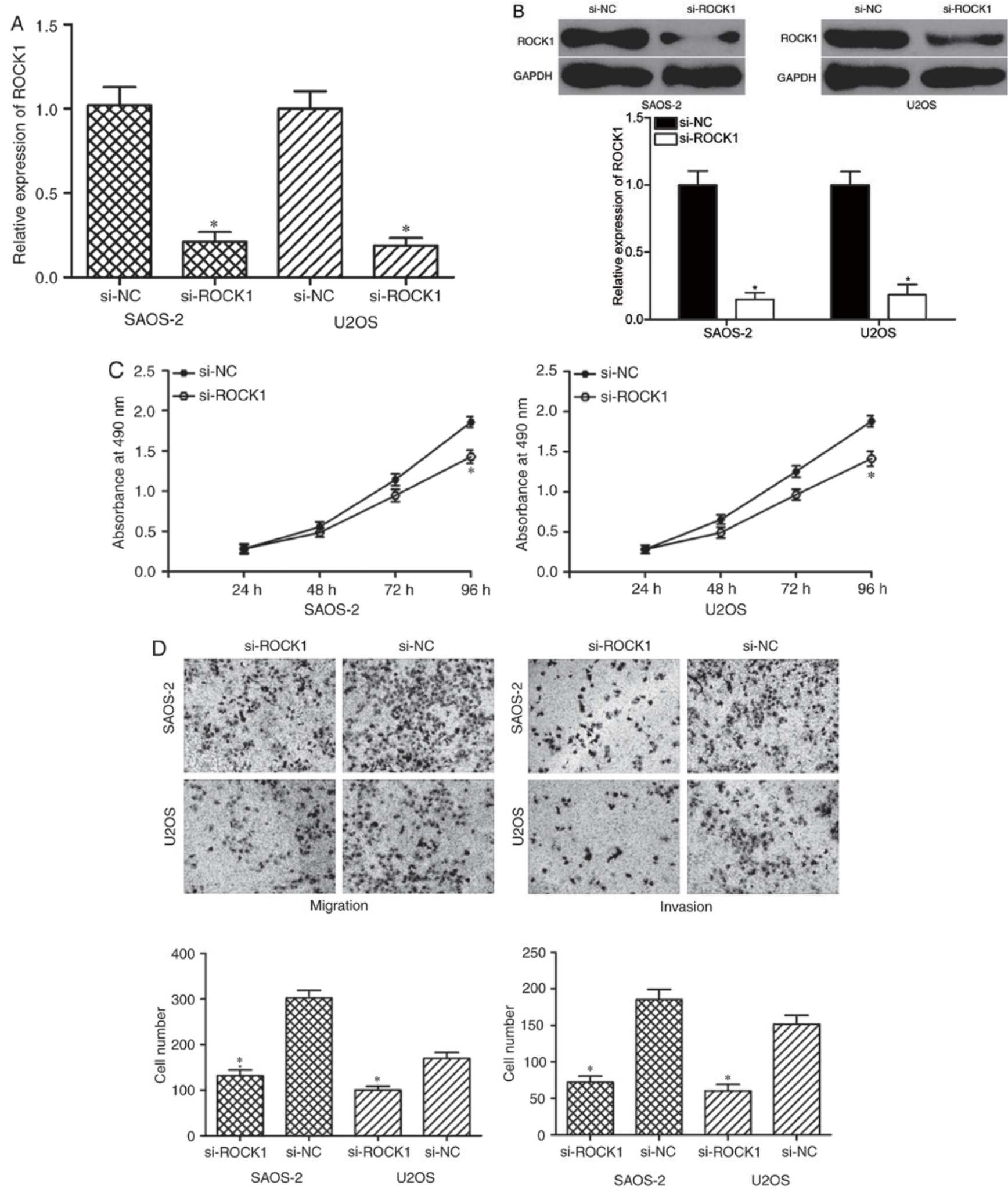

Figure 5. Downregulation of ROCK1 inhibits the proliferation, migration and invasion of SAOS-2 and U2OS cells. The SAOS-2 and U2OS cells were transfected with si-ROCK1 or si-NC. (A) mRNA and (B) protein expression levels of ROCK1 were downregulated in SAOS-2 and U2OS cells following transfection with si-ROCK1. (C) MTT assays were used to determine the proliferation of SAOS-2 and U2OS cells transfected with si-ROCK1 or si-NC. (D) Transwell migration and invasion assays were performed to evaluate the migration and invasion abilities of SAOS-2 and U2OS cells transfected with si-ROCK1 or si-NC (magnification, x200). "P<0.05, vs. si-NC. miR, microRNA; ROCK1, Rho-associated protein kinase 1; si-, small interfering RNA; NC, negative control.

suggest that ROCK1 can be investigated as a promising therapeutic target for the treatment of patients with OS.

In conclusion, the present study observed the downregulation of miR-129-5p in OS tissues and OS cell lines, whereas the ectopic expression of miR-129-5p suppressed tumor cell proliferation, migration and invasion. ROCK1 was identified as a direct target gene of miR-129-5p. The miR-129-5p/ROCK1 axis may be a promising therapeutic target for the treatment of OS. 


\section{References}

1. Ottaviani G and Jaffe N: The epidemiology of osteosarcoma Cancer Treat Res 152: 3-13, 2009.

2. Longhi A, Errani C, De Paolis M, Mercuri M and Bacci G: Primary bone osteosarcoma in the pediatric age: State of the art. Cancer Treat Rev 32: 423-436, 2006.

3. Jin J, Cai L, Liu ZM and Zhou XS: miRNA-218 inhibits osteosarcoma cell migration and invasion by down-regulating of TIAM1, MMP2 and MMP9. Asian Pac J Cancer Prev 14: 3681-3684, 2013.

4. Cho Y, Jung GH, Chung SH, Kim JY, Choi Y and Kim JD: Long-term survivals of stage IIb osteosarcoma: A 20-year experience in a single institution. Clin Orthop Surg 3: 48-54, 2011.

5. Bramer JA, van Linge JH, Grimer RJ and Scholten RJ: Prognostic factors in localized extremity osteosarcoma: A systematic review. Eur J Surg Oncol 35: 1030-1036, 2009.

6. Pierz KA, Womer RB and Dormans JP: Pediatric bone tumors: Osteosarcoma ewing's sarcoma and chondrosarcoma associated with multiple hereditary osteochondromatosis. J Pediatr Orthop 21: 412-418, 2001.

7. Poletajew S, Fus L and Wasiutynski A: Current concepts on pathogenesis and biology of metastatic osteosarcoma tumors. Ortop Traumatol Rehabil 13: 537-545, 2011 (In English, Polish).

8. Kong YW, Ferland-McCollough D, Jackson TJ and Bushell M: microRNAs in cancer management. Lancet Oncol 13: e249-e258, 2012.

9. Bartel DP: MicroRNAs: Genomics, biogenesis, mechanism, and function. Cell 116: 281-297, 2004

10. Kim VN: MicroRNA biogenesis: Coordinated cropping and dicing. Nat Rev Mol Cell Biol 6: 376-385, 2005.

11. Garzon R, Calin GA and Croce CM: MicroRNAs in cancer. Annu Rev Med 60: 167-179, 2009.

12. Liu J: Control of protein synthesis and mRNA degradation by microRNAs. Curr Opin Cell Biol 20: 214-221, 2008.

13. Carrington JC and Ambros V: Role of microRNAs in plant and animal development. Science 301: 336-338, 2003.

14. Tan X, Fan S, Wu W and Zhang Y: MicroRNA-26a inhibits osteosarcoma cell proliferation by targeting IGF-1. Bone Res 3 : 15033,2015

15. Zhao G, Dong L, Shi H, Li H, Lu X, Guo X and Wang J: MicroRNA-1207-5p inhibits hepatocellular carcinoma cell growth and invasion through the fatty acid synthase-mediated Akt/mTOR signalling pathway. Oncol Rep 36: 1709-1716, 2016.

16. Wu D, Niu X, Pan H, Zhou Y, Qu P and Zhou J: MicroRNA-335 is downregulated in bladder cancer and inhibits cell growth, migration and invasion via targeting ROCK1. Mol Med Rep 13: 4379-4385, 2016.

17. Croce CM: Causes and consequences of microRNA dysregulation in cancer. Nat Rev Genet 10: 704-714, 2009.

18. Yu Y, Zhao Y, Sun XH, Ge J, Zhang B, Wang X and Cao XC Down-regulation of miR-129-5p via the Twist1-Snail feedback loop stimulates the epithelial-mesenchymal transition and is associated with poor prognosis in breast cancer. Oncotarget 6 : 34423-34436, 2015 .

19. Jiang Z, Wang H, Li Y, Hou Z, Ma N, Chen W, Zong Z and Chen S: miR-129-5p is down-regulated and involved in migration and invasion of gastric cancer cells by targeting interleukin- 8 Neoplasma 63: 673-680, 2016.
20. Li J, Wang $\mathrm{H}$, Ke $\mathrm{H}$ and Ni S: miR-129 regulates MMP9 to control metastasis of non-small cell lung cancer. Tumour Biol 36 : 5785-5790, 2015.

21. Duan L, Hao X, Liu Z, Zhang Y and Zhang G: miR-129-5p is down-regulated and involved in the growth, apoptosis and migration of medullary thyroid carcinoma cells through targeting RET. FEBS Lett 588: 1644-1651, 2014.

22. Tan G, Cao X, Dai Q, Zhang B, Huang J, Xiong S, Zhang Yy, Chen W, Yang J and Li H: A novel role for microRNA-129-5p in inhibiting ovarian cancer cell proliferation and survival via direct suppression of transcriptional co-activators YAP and TAZ. Oncotarget 6: 8676-8686, 2015.

23. Karaayvaz M, Zhai $\mathrm{H}$ and Ju J: miR-129 promotes apoptosis and enhances chemosensitivity to 5-fluorouracil in colorectal cancer. Cell Death Dis 4: e659, 2013.

24. Livak KJ and Schmittgen TD: Analysis of relative gene expression data using real-time quantitative PCR and the 2(-Delta Delta C(T)) method. Methods 25: 402-408, 2001.

25. Liu X, Choy E, Hornicek FJ, Yang S, Yang C, Harmon D, Mankin $\mathrm{H}$ and Duan Z: ROCK1 as a potential therapeutic target in osteosarcoma. J Orthop Res 29: 1259-1266, 2011.

26. Lagos-Quintana M, Rauhut R, Yalcin A, Meyer J, Lendeckel W and Tuschl T: Identification of tissue-specific microRNAs from mouse. Curr Biol 12: 735-739, 2002.

27. Ma N, Chen F, Shen SL, Chen W, Chen LZ, Su Q, Zhang LJ, Bi J, Zeng WT, Li W, et al: MicroRNA-129-5p inhibits hepatocellular carcinoma cell metastasis and invasion via targeting ETS1. Biochem Biophys Res Commun 461: 618-623, 2015.

28. Zhai J, Qu S, Li X, Zhong J, Chen X, Qu Z and Wu D: miR-129 suppresses tumor cell growth and invasion by targeting PAK 5 in hepatocellular carcinoma. Biochem Biophys Res Commun 464: $161-167,2015$

29. Wang QY, Tang J, Zhou CX and Zhao Q: The down-regulation of miR-129 in breast cancer and its effect on breast cancer migration and motility. Sheng Li Xue Bao 64: 403-411, 2012 (In Chinese).

30. Luo J, Chen J and He L: mir-129-5p Attenuates Irradiation-Induced Autophagy and Decreases Radioresistance of Breast Cancer Cells by Targeting HMGB1. Med Sci Monit 21: 4122-4129, 2015.

31. Zhang C, Zhang S, Zhang Z, He J, Xu Y and Liu S: ROCK has a crucial role in regulating prostate tumor growth through interaction with c-Myc. Oncogene 33: 5582-5591, 2014.

32. Rossman KL, Der CJ and Sondek J: GEF means go: Turning on RHO GTPases with guanine nucleotide-exchange factors. Nat Rev Mol Cell Biol 6: 167-180, 2005.

33. Patel RA, Forinash KD, Pireddu R, Sun Y, Sun N, Martin MP, Schönbrunn E, Lawrence NJ and Sebti SM: RKI-1447 is a potent inhibitor of the Rho-associated ROCK kinases with anti-invasive and antitumor activities in breast cancer. Cancer Res 72: 5025-5034, 2012

34. Wang Y, Zhao W and Fu Q: miR-335 suppresses migration and invasion by targeting ROCK1 in osteosarcoma cells. Mol Cell Biochem 384: 105-111, 2013. 